\title{
Genome diversity in the genera Fructobacillus, Leuconostoc and Weissella determined by physical and genetic mapping
}

\author{
Ivo M. Chelo,† Líbia Zé-Zéł and Rogério Tenreiro
}

Correspondence

Ivo M. Chelo

imchelo@igc.gulbenkian.pt

Received 13 February 2009

Revised 19 October 2009

Accepted 2 November 2009
Centro de Biodiversidade, Genómica Integrativa e Funcional (BioFIG), Faculdade de Ciências da Universidade de Lisboa, Edificio ICAT, Campus da FCUL, Campo Grande, 1749-016 Lisboa, Portugal

Pulsed-field gel electrophoresis analysis of chromosomal single and double restriction profiles of 17 strains belonging to three genera of 'Leuconostocaceae' was done, resulting in physical and genetic maps for three Fructobacillus, six Leuconostoc and four Weissella strains. Ascl, I-Ceul, Notl and Sfil restriction enzymes were used together with Southern hybridization of selected probes to provide an assessment of genomic organization in different species. Estimated genome sizes varied from $1408 \mathrm{~kb}$ to $1547 \mathrm{~kb}$ in Fructobacillus, from $1644 \mathrm{~kb}$ to $2133 \mathrm{~kb}$ in

Leuconostoc and from $1371 \mathrm{~kb}$ to $2197 \mathrm{~kb}$ in Weissella. Other genomic characteristics of interest were analysed, such as oriC and terC localization and $r r n$ operon organization. The latter seems markedly different in Weissella, in both number and disposition in the chromosome. Comparisons of intra- and intergeneric features are discussed in the light of chromosome rearrangements and genomic evolution.

\section{INTRODUCTION}

The genera Fructobacillus, Leuconostoc and Weissella are composed of obligate heterofermentative bacterial species that, with species from the genus Oenococcus, constitute a single line of descent, the Leuconostoc group (Yang \& Woese, 1989; Martinez-Murcia \& Collins, 1990). Like other lactic acid bacteria (LAB) they are Gram-positive, nonspore-forming, inhabit nutrient-rich environments such as milk, meat, vegetable products and fermented drinks (Kandler \& Weiss, 1986) and have lactic acid as their main end product. The phylogenetic structure of these genera has been defined based on the analysis of 16S rRNA gene sequences (Martinez-Murcia \& Collins, 1990; Collins et al., 1993; Endo \& Okada, 2008) and in studies involving different molecular markers (Chelo et al., 2007; De Bruyne et al., 2007; Endo \& Okada, 2008). However, some taxonomic issues remain to be resolved, including the 'temporary' polyphyly of Leuconostoc as a result of the newly formed genus Fructobacillus (Endo \& Okada, 2008).

tPresent address: Instituto Gulbenkian de Ciência, Apartado 14, P2781-901 Oeiras, Portugal.

łPresent address: Centro de Estudos de Vectores e Doenças Infecciosas Dr Francisco Cambournac, Instituto Nacional de Saúde Dr Ricardo Jorge I.P., Av. Liberdade no. 5, 2965-575 Águas de Moura, Portugal.

Abbreviation: $L A B$, lactic acid bacteria.

Supplementary material is available with the online version of this paper.
As a supra-generic group, the Leuconostocs are phylogenetically related to Lactobacillus and Pediococcus (Vandamme et al., 1996; Makarova \& Koonin, 2007). Although there is currently high interest in LAB genomics (Klaenhammer et al., 2002, 2005; Makarova et al., 2006; Makarova \& Koonin, 2007) only three Leuconostoc genome sequences have been published so far, those of Oenococcus oeni PSU-1, Leuconostoc mesenteroides subsp. mesenteroides ATCC $8293^{\mathrm{T}}$ and Leuconostoc citreum KM20 (Makarova et al., 2006; Kim et al., 2008); four others (Leuconostoc mesenteroides, Leuconostoc gasicomitatum, Oenococcus oeni and Weissella paramesenteroides strains) are being sequenced (data from the ENTREZ Genome Project database at NCBI). The L. mesenteroides and $W$. paramesenteroides strains are relatively new additions to the ongoing sequencing projects and in the case of Weissella the only available information regarding genomic features such as chromosome size or number of rrn operons comes from the physical and genetic map of $W$. paramesenteroides DSM $20288^{\mathrm{T}}$ (Chelo et al., 2004). Perhaps due to this scarcity of information, comparative genomic analyses in the Leuconostoc group have been restricted to comparisons with species from other genera (Makarova et al., 2006; Makarova \& Koonin, 2007; Marcobal et al., 2007) or are limited to a single species (Zé-Zé et al., 2000, 2008).

In this study we analysed the chromosomes of 17 strains of the genera Fructobacillus, Leuconostoc and Weissella by restriction with $A s c \mathrm{I}, \mathrm{I}-\mathrm{CeuI}$, NotI and SfiI and electrophoretic separation by PFGE. Together with Southern 
hybridization of selected probes we were able to construct 13 new physical and genetic maps on which genomic features of interest were localized. A map generated by in silico analysis of the L. citreum KM20 chromosome was also included in this study. The comparison of different strains in each genus, representing different species, has enabled us to unveil the main types of macrogenomic evolutionary events that shaped the diversification of this Leuconostoc group.

\section{METHODS}

Bacterial strains and growth conditions. All strains studied (see Table 1) were grown in MRS medium, $\mathrm{pH} 6.5$, at $30{ }^{\circ} \mathrm{C}$ without shaking (with the exception of Leuconostoc gelidum DSM $5578^{\mathrm{T}}$, which was grown at $22{ }^{\circ} \mathrm{C}$ ). For Fructobacillus strains, the growth medium was supplemented with D-fructose at $2 \%$. Escherichia coli JM109 and XL-1 Blue MRF' (Stratagene) were grown at $37^{\circ} \mathrm{C}$ in LB broth, supplemented with $100 \mu \mathrm{g}$ ampicillin $\mathrm{ml}^{-1}$ when required.

Isolation and cleavage of chromosomal DNA, and DNA fragment nomenclature. Intact genomic DNA was prepared in agarose plugs and single or double digested with the restriction enzymes AscI, I-CeuI, NotI and SfiI as previously described (Tenreiro et al., 1994; Zé-Zé et al., 1998, 2000). Restriction fragments produced by digestion with a single enzyme are indicated by the initial letter of the endonuclease. Nomenclature for fragments resulting from double digestion, co-migrating fragments and hybridization results (see the supplementary material available with the online version of this paper) follow Chelo et al. (2004). DNA extraction and purification was done using the method described by Pitcher et al. (1989). Some DNA fragments were recovered after gel electrophoresis using the Jet Quick Gel extraction spin kit (Genomed). In the case of $L$. citreum KM20 the relative localization of restriction sites was done with pDRAW32 1.0 (AcaClone).

PFGE. PFGE was carried out in the Gene Navigator system (Pharmacia) with contour-clamped homogeneous electric field (CHEF) as previously described (Zé-Zé et al., 1998). A different run was also used for the separation of fragments ranging from 4 to $50 \mathrm{~kb}$. With a total time of $12 \mathrm{~h}$ at $260 \mathrm{~V}$, this run consists of steps of $4 \mathrm{~h}$ with a pulse time of $0.8 \mathrm{~s}, 4 \mathrm{~h}$ with a pulse time of $1 \mathrm{~s}$, and $4 \mathrm{~h}$ with a pulse time of $1.2 \mathrm{~s}$. Staining was done with ethidium bromide or SYBR Green. The mean size of each fragment was estimated from at least two (fragments larger than $1000 \mathrm{~kb}$ ) or six (fragments smaller than $1000 \mathrm{~kb}$ ) runs by linear interpolation with two or more flanking size standards using KODAK 1D 2.0 software. Lambda ladder, LowRange PFG Markers (New England Biolabs), DNA Size Markers Sschizosaccharomyces pombe chromosomal DNA and $2.5 \mathrm{~kb}$ Molecular Ruler (Bio-Rad) - were used as molecular mass standards as well as intact chromosomes of Saccharomyces cerevisiae (also from Bio-Rad).

PCR conditions and plasmid construction. Most PCR conditions and plasmids used in this work have been previously described (Chelo et al., 2004). A complete list of primers and amplification conditions can be found in Supplementary Table S4.

Table 1. Strains used and genome characteristics

\begin{tabular}{|c|c|c|c|c|c|c|}
\hline Strain & \multicolumn{4}{|c|}{ No. of fragments generated by restriction } & $\begin{array}{c}\text { Estimated } \\
\text { chromosome } \\
\text { size in } \mathbf{k b}^{\star}\end{array}$ & $\begin{array}{c}\text { Map (no. } \\
\text { of enzymes) }\end{array}$ \\
\hline Leuconostoc mesenteroides subsp. mesenteroides CECT $219^{\mathrm{T}} \dagger$ & 2 & 4 & 5 & 5 & $2032(78)$ & Yes $(4)$ \\
\hline Leuconostoc mesenteroides subsp. cremoris CECT $872^{\mathrm{T}}$ & 6 & 4 & 14 & NA & $1644(16)$ & Yes $(3)$ \\
\hline Leuconostoc citreum CECT $4025^{\mathrm{T}}$ & 5 & 5 & 6 & 4 & $1850(33)$ & Yes $(4)$ \\
\hline Leuconostoc gelidum DSM $5578^{\mathrm{T}}$ & 6 & 4 & 5 & 6 & $1953(83)$ & Yes $(4)$ \\
\hline Leuconostoc pseudomesenteroides CECT $4027^{\mathrm{T}}$ & 12 & 4 & 7 & 3 & $2133(34)$ & Yes $(4)$ \\
\hline Leuconostoc fallax DSM $20189^{\mathrm{T}}$ & 0 & 4 & 0 & 2 & $1648 \pm 45$ & No \\
\hline Fructobacillus ficulneus DSM $13613^{\mathrm{T}}$ & 4 & 5 & 15 & $>35$ & $1547(5)$ & Yes $(3)$ \\
\hline Fructobacillus pseudoficulneus LC51 $^{\mathrm{T}}$ & 4 & 4 & 23 & $>30$ & $1408(13)$ & Yes (3) \\
\hline Weissella confusa DSM $20196^{\mathrm{T}}$ & 13 & 8 & 10 & 13 & $2197(16)$ & Yes $(3)$ \\
\hline Weissella kandleri CECT $4307^{\mathrm{T}}$ & 8 & 6 & 7 & 4 & $1371(10)$ & Yes $(4)$ \\
\hline Weissella halotolerans CECT $573^{\mathrm{T}}$ & $>15$ & NA & $>25$ & $>35$ & NA & No \\
\hline Weissella viridescens DSM $20410^{\mathrm{T}}$ & 2 & 7 & 9 & 9 & $1520(22)$ & Yes $(4)$ \\
\hline
\end{tabular}

NA, Non-available. Restriction profiles always presented several fragments resulting from incomplete digestion, or fragments of very low intensity. ${ }^{\star}$ Deviations from the mean values are given by the maximal size deviation $(\mathrm{kb})$ from the observed genome size to the mapped size when a map was obtained (values in parentheses), or in the case of L. fallax as the SEM of the different enzymes.

†Although genomic maps of these strains have already been published (Chelo et al., 2004; Makarova et al., 2006) they are included here for comparative purposes. 
DNA probes. All probes used in this work (see Table 2 for probes used and some of their characteristics) were labelled with digoxigenin using PCR Dig labelling mix (Roche). Transposase B, Permease (AA) and Transport $\mathrm{ABC}$ probes were used as linking clones, since they contain restriction sites of the enzymes used in this study (NotI site for Transposase B and AscI sites for the others). Sequences for these probes were obtained from the US DOE Joint Genome Institute (JGI) in an early version of the sequencing project of L. mesenteroides subsp. mesenteroides ATCC $8293^{\mathrm{T}}$ [Transposase B corresponds to locus tag LEUM_A02, Permease (AA) to LEUM_1791 and Transport ABC to LEUM_0693].

In silico analysis. The localization of markers in L. citreum KM20 was done using the sequenced genome annotation when possible or by BLAST analysis (Altschul et al., 1990). For the comparison of L. mesenteroides subsp. mesenteroides CECT $219^{\mathrm{T}}$ and L. citreum KM20 strains, TBLASTX alignments were done with a cut-off value of $10^{-4}$ in the WebACT site (http://www.webact.org/WebACT/ home).

ITS assignment. Identification of the different sets of tRNA genes in the ITS regions of specific rrn operons of Weissella strains was based on explicit I-CeuI fragment PCR amplification. In Fructobacillus and Leuconostoc strains genomic DNA amplification always gave rise to a single band of the size expected for tRNA ${ }^{\text {Ala }}$, as previously seen for most strains (Endo \& Okada, 2008). Detailed explanation of the method can be found in Chelo et al. (2004).

\section{RESULTS}

\section{Macrorestriction fragments and genome sizes of Fructobacillus, Leuconostoc and Weissella strains}

The numbers of restriction fragments generated with AscI, NotI, SfiI and the homing endonuclease I-CeuI as well as the estimated genome sizes are presented in Table 1 . Genome sizes are averages of estimates with the different enzymes (two to four enzymes) and they match genomemapped sizes when a physical map was obtained. The fully assembled genome sequence of $L$. mesenteroides subsp. mesenteroides ATCC $8293^{\mathrm{T}}$ has become available, revealing a chromosome size of $2038 \mathrm{~kb}$ (Makarova et al., 2006; data available at the NCBI and JGI databases). This strain corresponds to the strain CECT $219^{\mathrm{T}}$ that was analysed in this study but since this map was built without the prior knowledge of the assembled sequence, we chose to present it for comparative purposes. In this way, the error inherent in our approach is also reflected in this map, although we have a priori knowledge of its suitability.

The analysis of the number of restriction fragments generated in this study reveals diverse results. The number

Table 2. DNA sequences used as probes in this study

\begin{tabular}{|c|c|c|c|c|}
\hline Probe & Gene (function/description)* & $\begin{array}{l}\text { Approx. fragment } \\
\text { size }(\mathbf{k b})\end{array}$ & $\begin{array}{c}\mathrm{G}+\mathrm{C} \text { content } \\
(\mathrm{mol} \%)\end{array}$ & Reference for probes \\
\hline$r r s$ & Small-subunit rRNA [LEUM_r0019] & 1.5 & 51.4 & Chelo et al. (2004) \\
\hline$r r l$ & Large-subunit rRNA & 1.1 & 50.3 & Chelo et al. (2004) \\
\hline$d n a A$ & $\begin{array}{l}\text { Chromosomal replication initiator protein DnaA } \\
\text { [LEUM_0001] }\end{array}$ & 0.7 & 37.7 & Chelo et al. (2004) \\
\hline gyrB & DNA gyrase subunit B [LEUM_0005] & 1.8 & 45.6 & Chelo et al. (2004) \\
\hline rpoC & DNA-directed RNA polymerase subunit $\beta^{\prime}$ [LEUM_1824] & 1.4 & 42.3 & Chelo et al. (2004) \\
\hline$d n a K$ & Chaperone protein DnaK [LEUM_1347] & 0.6 & 51.4 & Chelo et al. (2004) \\
\hline recA & RecA protein [LEUM_0584] & 0.25 & 49.6 & Chelo et al. (2004) \\
\hline GACA3 & Undetermined, no coding & 3.5 & 43.8 & Chelo et al. (2004) \\
\hline intC8 & 3-Oxoacyl reductase; possible RNA methyltransferase $\dagger$ & 2.8 & 34.1 & Chelo et al. (2004) \\
\hline $\operatorname{tgt}, \mathrm{clp} B$ & $\begin{array}{l}\text { Queuine tRNA-ribosyltransferase/CLPB ATP-binding } \\
\text { protein [LEUM_0376] }\end{array}$ & 6 & 42.2 & Zé-Zé et al. (2000) \\
\hline harosynth & $\begin{array}{l}\text { 3-Phosphoshikimate 1-carboxyvinyltransferase } \\
\text { [LEUM_1164] }\end{array}$ & 0.8 & 40.9 & Chelo et al. (2004) \\
\hline hetpsynth & CTP synthase [LEUM_0519] & 0.6 & 41.7 & Chelo et al. (2004) \\
\hline hred, htransp & Aldo-keto reductase; putative transposase $\dagger$ & 1.4 & 32.8 & Chelo et al. (2004) \\
\hline hpolC & $\begin{array}{l}\text { DNA polymerase III catalytic subunit, PolC type } \\
\text { [LEUM_0689] }\end{array}$ & 0.4 & 43.0 & Chelo et al. (2004) \\
\hline $\mathrm{h} r p o A$ & DNA-directed RNA polymerase subunit $\alpha$ [LEUM_0221] & 0.4 & 42.0 & Chelo et al. (2004) \\
\hline h5' nucl & Putative 5-nucleotidase $\dagger$ & 0.7 & 39.8 & Chelo et al. (2004) \\
\hline Permease (AA) & Amino acid transporter [LEUM_1791] & 1.4 & 38.6 & This study \\
\hline Transport $\mathrm{ABC}$ & $\begin{array}{l}\text { Uncharacterized ABC-type transport system, } \\
\text { nucleoside-binding protein [LEUM_0693] }\end{array}$ & 0.65 & 37.9 & This study \\
\hline$\alpha$ ATPase & ATP synthase F1 subcomplex $\alpha$ subunit [LEUM_1871] & 1.1 & 43.4 & This study \\
\hline Transposase B & Transposase B, hypothetical protein [LEUM_A02] & 0.6 & 38.0 & This study \\
\hline
\end{tabular}

${ }^{*}$ When available, locus tags are given for probes that hybridized to the L. mesesenteroides subsp. mesenteroides genome.

$\dagger$ Possible function of the DNA sequences was obtained by BLAST searches (BLASTX or BLASTP) as described previously (Chelo et al., 2004). 
of AscI, NotI and SfiI restriction fragments shows considerable variation, ranging from 2 to more than 15 for AscI, 0 to more than 25 for NotI and 2 to more than 35 for Sfil. The high number of restriction sites often prevents their use for mapping purposes. Taking into consideration the different degrees of evolutionary proximity of most of the strains used in this study (Chelo et al., 2007), we can also see that in some cases a high proportion of the variation can be found in closely related strains. This is the case for the number of AscI fragments in L. citreum CECT 4018 (15 fragments) and $L$. citreum CECT $4025^{\mathrm{T}}$ (5 fragments) or NotI fragments in L. mesenteroides subsp. cremoris CECT $872^{\mathrm{T}}$ (14 fragments) and L. mesenteroides subsp. mesenteroides (5 fragments). A similar situation is seen for the Not Iragments in the Weissella strains. These show a much greater difference between $W$. confusa DSM $20196^{\mathrm{T}}$ (10 fragments) and $W$. hellenica DSM $7378^{\mathrm{T}}$ (2 fragments) than between $W$. confusa DSM $20196^{\mathrm{T}}$ and $W$. viridescens DSM $20410^{\mathrm{T}}$ (9 fragments), which are evolutionarily less related. In contrast to this inconsistency of fragment number and evolutionary relatedness, Fructobacillus strains represent a case where the variation is reduced.

The number of I-CeuI-generated fragments, indicative of the number of $r r n$ operons in the chromosome, reveals a different situation. In addition to an overall smaller degree of variation in comparison with the other restriction profiles, a distinction between Weissella and the other two genera can be readily made. In both Fructobacillus and Leuconostoc, restriction with I-CeuI seems to consistently give four fragments, with the exception of $L$. citreum CECT $4025^{\mathrm{T}}$ and Fructobacillus ficulneus DSM $13613^{\mathrm{T}}$, whereas in Weissella this number varies between six and eight.

Chromosome sizes also provide an interesting view of the degree of variation in these genomes. The highest and lowest values can be found in W. confusa DSM $20196^{\mathrm{T}}$ $(2197 \mathrm{~kb})$ and $W$. kandleri CECT $4307^{\mathrm{T}}(1371 \mathrm{~kb})$ respectively; Fructobacillus and Leuconostoc show smaller ranges of variation - $1408 \mathrm{~kb}$ to $1547 \mathrm{~kb}$ in Fructobacillus and $1644 \mathrm{~kb}$ to $2133 \mathrm{~kb}$ in Leuconostoc. This is consistent with a later diversification between the Fructobacillus and Leuconostoc genera. As for the number of some restriction sites, a large variation in chromosome size is observed even in closely related strains, for example L. mesenteroides subsp. mesenteroides CECT $219^{\mathrm{T}}$ and L. mesenteroides subsp. cremoris CECT $872^{\mathrm{T}}$, which differ by about $21 \%$ (considering a mean chromosome size of $1838 \mathrm{~kb}$ ).

\section{Comparison of physical and genetic maps in Fructobacillus and Leuconostoc}

Given both the historical association of Fructobacillus and Leuconostoc species and the presence of many common characteristics in these genera, their genomic features are described together. The analysis of double digestions together with Southern hybridization of selected probes (see Table 2 and supplementary material) gives the relative localization of many restriction sites and genes, allowing the construction of the physical and genetic maps in Figs 1, 2 and 3. Connecting lines between successive maps identify the position of the same marker in different chromosomes and indicate whether this marker is localized in a conserved region (blue line) or in a region that might have been subject to an inversion event (red line). If no such assessments can be made the connecting line is grey. This classification was obtained by the following procedure. Considering the linear order of shared markers in two circular chromosomes as ordered vectors $X=x_{1} \ldots x_{\mathrm{n}}$ and $Y=y_{1} \ldots y_{\mathrm{n}}$, a marker $x_{\mathrm{i}}=y_{\mathrm{j}}$ is said to be in a conserved region (blue) if $x_{i-1}=y_{j-1}$ or $x_{i+1}=y_{j+1}$. Otherwise, if $x_{i-1}=y_{j+1}$ or $x_{i+1}=y_{j-1}$ we consider that an inversion (red) has occurred. Since this process is applied to each shared marker it is possible that an inversion line is plotted for a single marker (as in Figs 1 and 3). When there are alternative mapping possibilities these rules can also be applied as long as the final result is always the same, as in the comparison between F. ficulneus DSM $13613^{\mathrm{T}}$ and F. pseudoficulneus $\mathrm{LC} 51^{\mathrm{T}}$ in Fig. 1. When several markers are allocated to the same fragment, they do not provide relative information and thus we assume they cannot support or contradict assessments made with the other markers (for instance, in the comparison of L. gelidum DSM $5578^{\mathrm{T}}$ with F. ficulneus DSM $13613^{\mathrm{T}}$ in Fig. 1, rpoC is considered to be in a conserved region since $\operatorname{tgt} / \operatorname{clp} B$ provides a valid adjacent marker).

Data including restriction profiles, hybridization results and circular maps required for mapping purposes are given for a representative strain of each genus in the supplementary material (Supplementary Files S1-S3; data for other strains are available from the authors on request). The probes used should provide a good estimate of genomic organization dynamics in the strains studied, as revealed by their consistency with nearby markers obtained from whole genome sequencing (Supplementary Fig. S4).

In Fig. 1 a comparison of linear maps of type strains of Fructobacillus spp. and Leuconostoc spp. is provided. Most of the distinctive features that can be evaluated at this level seem to be well conserved. The origin of transcription oriC (assessed by the position of the dnaA and gyrB markers) is surrounded by $r r n$ operons that are transcribed divergently from it. The distribution of the $r r n$ operons is largely circumscribed around oriC, being localized in $22.1 \%$ (in $L$. gelidum DSM $5578^{\mathrm{T}}$ ) to $42.4 \%$ (in F. fructosum DSM $20439^{\mathrm{T}}$ ) of the chromosome. It is noteworthy that when $r r$ operons occupy a more significant part of the chromosome (in Fructobacillus strains), the absolute range (around $600 \mathrm{~kb}$ ) is similar in all chromosomes. Although rrn operons are usually equally distributed on both sides of oriC there are a few exceptions such as in F. pseudoficulneus $\mathrm{LC}^{\mathrm{T}} 1^{\mathrm{T}}$ and F. fructosus DSM $20349^{\mathrm{T}}$, where they have a $3: 1$ configuration (three on one side of oriC and one on the other), or in $L$. gelidum DSM $5578^{\mathrm{T}}$, where the asymmetry is the greatest, in a 4:0 configuration. 


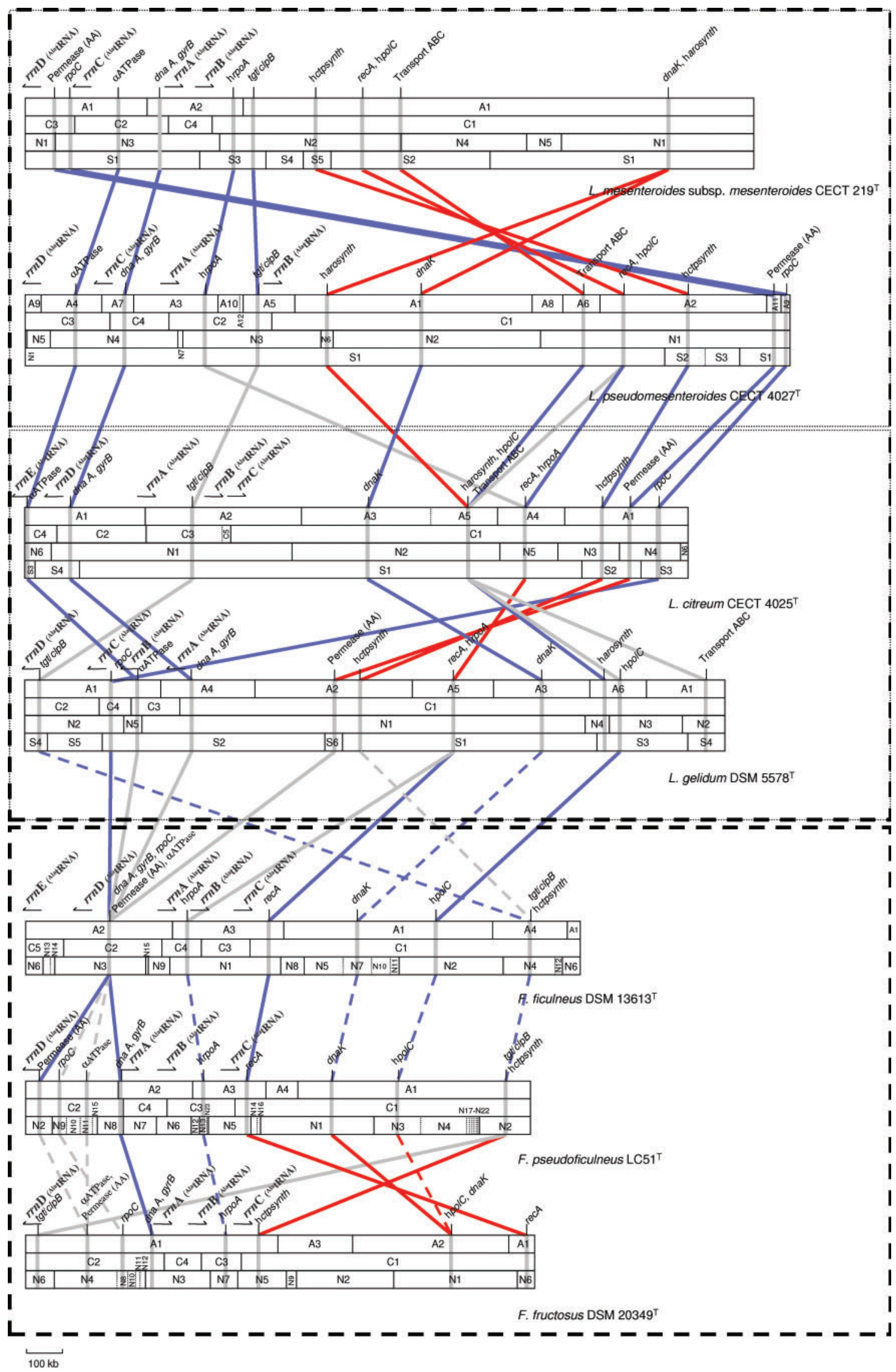


Fig. 1. Physical and genetic maps of type strains of Leuconostoc spp. and Fructobacillus. Restriction sites for Ascl, I-Ceul, Notl and Sfil are indicated. The circular genomes (see supplementary material) were linearized from the most distant $r r n$ operon regarding $d n a A$ that was localized in the counter-clockwise direction. Genetic markers are placed in the median position of the smallest fragment where they hybridized. Connecting lines between maps are colour-coded as follows: markers in conserved genomic regions in blue; markers in genomic regions subjected to inversions in red; and markers in other regions in grey (see text for details). Dashed lines in restriction maps indicate that the relative order of flanking fragments is unknown. Lines connecting markers in such regions are also dashed. The black dashed rectangles around maps limit comparisons within different genera.

The presence and order of genetic markers in these chromosomes also indicates a high degree of maintenance of genomic organization. Of the 13 markers analysed in these strains only Transport $\mathrm{ABC}$ and harosynth are not present in all genomes, being absent in the Fructobacillus strains. A few markers are consistently found close to oriC. That is the case for $\alpha$ ATPase, Permease (AA) and also rpoC. On the right side of the linear maps, where the terminus of replication terC is probably localized (corresponding to a $180^{\circ}$ position in the circular maps) it is possible to find the presence of the dnaK marker. The presence of many markers in conserved regions is however reduced in comparisons of strains belonging to different clusters. Still, it should be noted that many grey connecting lines are just the result of the impossibility of separating markers in some chromosomes. Probably the most noteworthy feature concerning genomic rearrangements is the presence of large inversions. This is clearly seen in the comparisons of $L$. mesenteroides subsp. mesenteroides CECT $219^{\mathrm{T}}$ with $L$. pseudomesenteroides CECT $4027^{\mathrm{T}}$ and of F. pseudoficulneus $\mathrm{LC}^{\mathrm{T}}{ }^{\mathrm{T}}$ with $F$. fructosus DSM $20349^{\mathrm{T}}$, but it might also have occurred in the evolution of L. citreum CECT $4025^{\mathrm{T}}$ or $L$. gelidum DSM $5578^{\mathrm{T}}$. From what is seen in the first two cases these inversions involve a large part of the chromosome, about $50 \%$, and are symmetrical with respect to oriC. Although the analysis of the sequenced genomes of $L$. mesenteroides subsp. mesenteroides CECT $219^{\mathrm{T}}$ and L. citreum KM20 (Supplementary Fig. S4) cannot be fully comparable to that presented in Fig. 1 it is nevertheless consistent with the presence of a generally conserved genomic backbone and genomic inversions.

At a more restricted level, the presence of rearrangements can also be inferred even if their exact nature is unknown. This is the case for the linear order of rpoC-rrn operon$\alpha$ ATPase-rrn operon- $d n a A$, gyrB that can be seen not only in L. pseudomesenteroides CECT $4027^{\mathrm{T}}$ but also in $L$. citreum CECT $4025^{\mathrm{T}}$ and L. gelidum DSM $5578^{\mathrm{T}}$ and that is changed in L. mesenteroides subsp. mesenteroides CECT $219^{\mathrm{T}}$.

An evaluation of genomic organization at the intra-specific level can be made from Fig. 2, which shows linear maps of $L$. mesenteroides and L. citreum strains; the linear map of the sequenced L. citreum KM20 (Kim et al., 2008) is also included in this figure. Both in the comparison of $L$. mesenteroides subsp. mesenteroides CECT $219^{\mathrm{T}}$ with $L$. mesenteroides subsp. dextranicum CECT $912^{\mathrm{T}}$ and in the comparison of L. citreum CECT $4025^{\mathrm{T}}$ and L. citreum
KM20 the order of markers is highly conserved. It must be noted that the apparent discrepancy in the order of the hpolC, Transport ABC, harosynth and dnaK markers in the two L. citreum strains may just be the result of a misplacement of fragments A3 and A5 in L. citreum CECT $4025^{\mathrm{T}}$ (in cases of lack of resolution the fragments are arbitrarily displayed in descending order of size, from left to right in the linear map). In contrast, and even taking into account possible inaccuracies, $L$. mesenteroides subsp. cremoris CECT $872^{\mathrm{T}}$ and L. citreum CECT $4018^{\mathrm{T}}$ show a greater number of differences in the order of markers. These indicate the occurrence of genomic rearrangements, which cannot be described in a simple way. In the case of the L. mesenteroides subsp. cremoris strain the differences in the order of markers from the other two L. mesenteroides strains would require at least two independent transposition events [involving recA and Permease (AA)] to be invoked. Nevertheless, differences in the disposition of the $r p o C$, $\alpha$ ATPase and $d n a A / g y r B$ and $r r n$ operons imply that genomic rearrangements also occurred that changed the order of these markers and possibly the direction of gene transcription. In this respect, it should be pointed out that the L. mesenteroides subsp. cremoris strain seems to be more similar to the type strains of other close Leuconostoc species, possibly revealing the ancestral order of these markers. The comparison of the three L. mesenteroides maps is also interesting as regards genomic size. There are marked differences in the sizes of the chromosomes (Fig. 2), which seem to be circumscribed to the region flanked by the Transport $\mathrm{ABC}$ and dnaK markers, in contrast to the region surrounding oriC, where genomic size is more conserved.

Another significant result is the ubiquitous presence of the Transposase marker in the chromosomes of two $L$. mesenteroides strains and especially $L$. citreum CECT 4018. This marker is a linking clone (has an AscI site) in L. mesenteroides subsp. mesenteroides CECT $219^{\mathrm{T}}$, where it is present in a plasmid (Makarova et al., 2006; this work). The co-occurrence of an increased number of AscI sites and hybridization targets for the Transposase marker in those chromosomes is a strong indication of the maintenance of that AscI restriction site. The propagation of a transposase thus becomes the most likely explanation for the high number of AscI fragments in the chromosomes of $L$. mesenteroides subsp. dextranicum CECT $912^{\mathrm{T}}$, L. mesenteroides subsp. cremoris CECT $872^{\mathrm{T}}$ and L. citreum CECT 4018. 


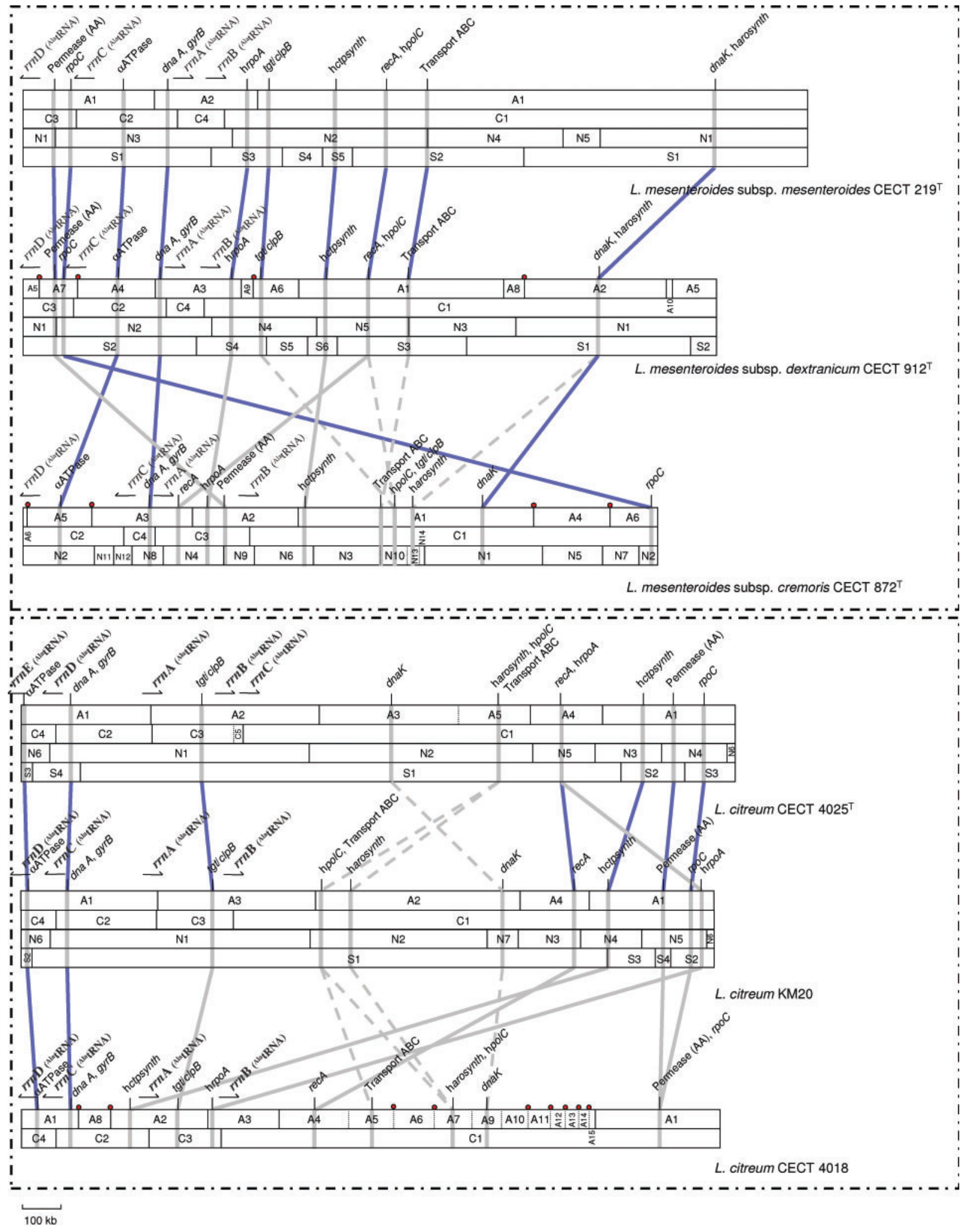

Fig. 2. Intra-specific comparison of physical and genetic maps of Leuconostoc strains. Restriction sites for Ascl, I-Ceul, Notl and Sfil are indicated. The circular genomes (see Supplementary material) were linearized from the most distant $r r n$ operon regarding $d n a A$ that was localized in the counter-clockwise direction. Genetic markers are placed in the median position of the smallest fragment where they hybridized. Small red circles indicate the location of the Transposase marker. Connecting lines between maps are colour-coded as in Fig. 1. Dashed lines in restriction maps indicate that the relative order of flanking fragments is unknown. Lines connecting markers in such regions are also dashed. The black dashed rectangles around maps limit comparisons within different species. 


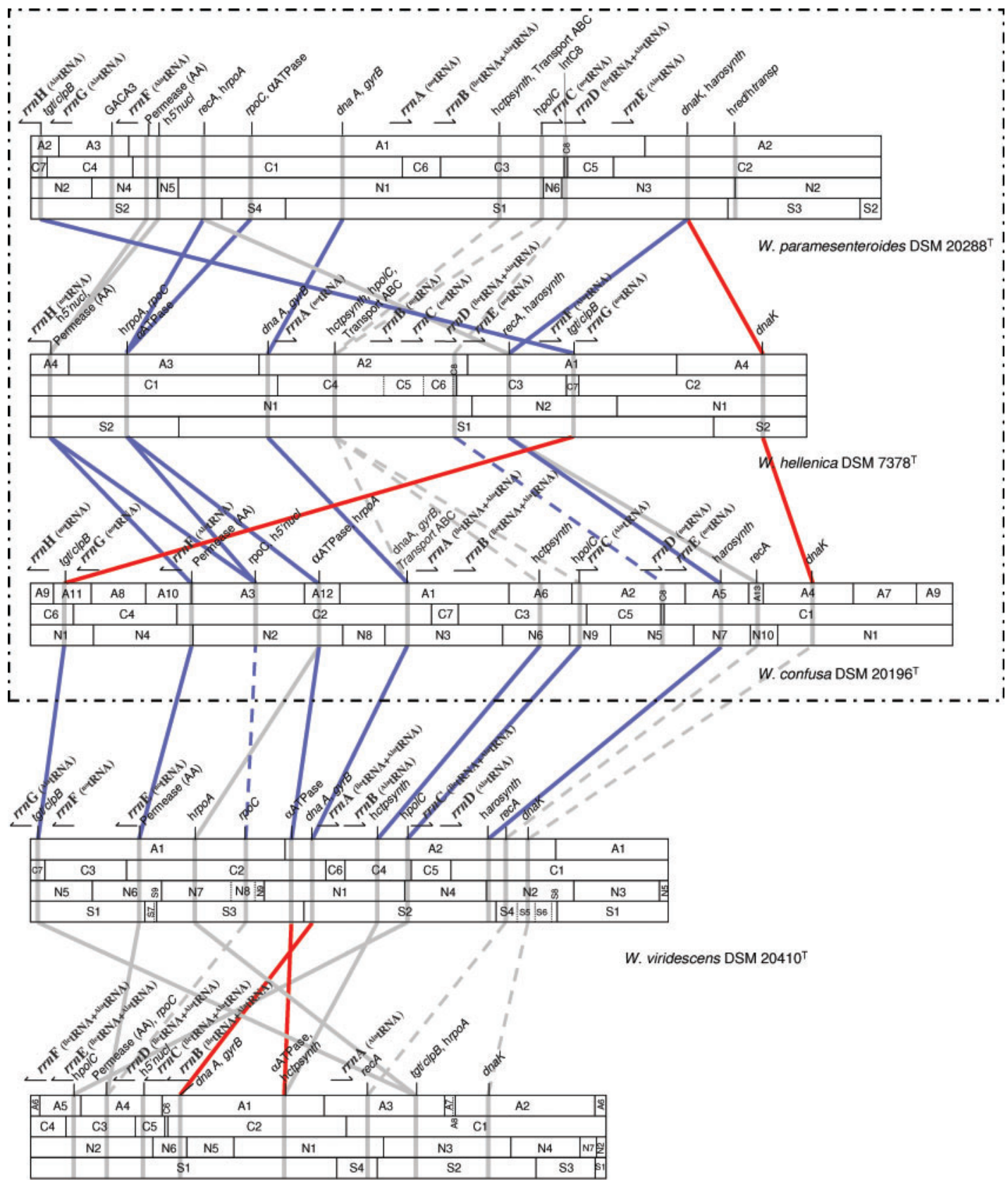

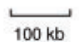

W. kandleri CECT $4307^{\top}$

Fig. 3. Physical and genetic maps of type strains of Weissella spp. Restriction sites for Ascl, l-Ceul, Notl and Sfil are indicated. The circular genomes (see supplementary material) were linearized from the most distant $r r n$ operon regarding $d n a A$ that was localized in the counter-clockwise direction. Genetic markers are placed in the median position of the smallest fragment where they hybridized. Connecting lines between maps are colour-coded as in Fig. 1. Dashed lines in restriction maps indicate that the relative order of flanking fragments is unknown. Lines connecting markers in such regions are also dashed. The black dashed rectangle around maps limits the monophyletic group referred to as the $W$. paramesenteroides group.

\section{Comparison of physical and genetic maps in Weissella}

When comparing the genomic organization of Weissella strains (Fig. 3) with those described for Fructobacillus and
Leuconostoc strains (Figs 1 and 2) it is possible to see that only a few general characteristics are preserved. Once again the ribosomal operons are transcribed divergently from oriC and the markers $\alpha$ ATPase and rpoC are consistently found close to dnaA. dnaK again appears to be located in 
the same region as terC. Apart from these conserved features many differences are evident. Concerning rrn operon organization in Weissella, rrn copy number can vary from 6 in $W$. kandleri CECT $4307^{\mathrm{T}}$ to 8 in strains of the $W$. paramesenteroides cluster. Furthermore, rrn operons are more dispersed in the chromosomes of Weissella. In $W$. kandleri CECT $4307^{\mathrm{T}}$ they are present in about $55 \%$ of the chromosome and in W. confusa DSM $20196^{\mathrm{T}}$ this value increases to $69 \%$. As part of this same organization oriC now lies in a large I-CeuI fragment, in contrast to both Fructobacillus and Leuconostoc maps (Fig. 1), even if it still seems to be located close to a rrn operon. In Wiessella strains, the rrn operons are also unlike Fructobacillus or Leuconostoc rrn operons, in which they can have different tRNAs coded in their ITS regions. However, this characteristic fails in its possible use to match rrn operons in different strains since even clearly homologous $\mathrm{rr}$ operons can have different tRNA sets (see rrn operons flanking the intC8 marker in Fig. 3), possibly as a result of intra-genomic recombination.

As previously seen, the genomic organization can be more different in more evolutionarily closely related strains than in more distant ones. The $W$. paramesenteroides DSM $20288^{\mathrm{T}}$ chromosome appears to be more different from the $W$. hellenica DSM $7378^{\mathrm{T}}$ chromosome than from that of $W$. confusa DSM 20196 $6^{\mathrm{T}}$, to which it is less related (Chelo et al., 2007). This is most evident in the order of rrn operons. Both the $r r n$ disposition around oriC $(3: 5)$ and the relative distance between operons are more similar in the less related strains. In the evolution of the W. hellenica DSM $7378^{\mathrm{T}}$ line it is also possible that an inversion involving tgt/ $c l p B$ and dnaK markers has occurred. However, looking only at the comparison with the $W$. paramesenteroides strain the possibility of a transposition of the dnaK marker region cannot be discarded. When the less related $W$. viridescens DSM $20410^{\mathrm{T}}$ and W. kandleri CECT $4307^{\mathrm{T}}$ are compared with the strains of the $W$. paramesenteroides cluster (more particularly with W. confusa DSM 20196 ${ }^{\mathrm{T}}$ ) the degree of conservation of gene order in the case of $W$. viridescens DSM $20410^{\mathrm{T}}$ is remarkable, especially taking into account the differences in chromosome size. The region between the $r r n G$ operon in $W$. viridescens DSM $20410^{\mathrm{T}}$ (corresponding to the $r r n H$ operon in W. confusa DSM 20196 ${ }^{\mathrm{T}}$ ) and $r r n A$ appears to have been less subject to changes in size than the rest of the chromosome. The chromosome of $W$. kandleri CECT $4307^{\mathrm{T}}$, which is the smallest of this study, also reveals a great number of differences, making it difficult to distinguish the presence of any rearrangements or even the correct placement of the map for comparative purposes.

\section{DISCUSSION}

The construction of physical and genetic maps, based on PFGE analysis of macrorestriction fragments and hybridization of genetic markers of several Fructobacillus, Leuconostoc and Weissella strains, enabled the comparison of genomic organization at different taxonomic levels in the Leuconostoc group of LAB. One of the first findings is that evolutionary relatedness is generally well reflected at the level of genomic organization in terms of the degree of synteny or maintenance of genomic features such as $\mathrm{rr}$ operon organization. Thus, surprisingly, it is possible that even close strains show what seem to be marked differences, as in the case of the number of restriction fragments generated by rare-cutting enzymes. However, the strains used in this study show that large differences in the number of AscI sites and to a lesser extent of NotI or SfiI sites may not be significant to the overall chromosome organization and are thus meaningless at the level of genomic comparisons. It was possible to associate the presence of transposable elements with the increase in AscI sites in several Leuconostoc strains. Interestingly, the Transposase marker that was used for this purpose was first found in a plasmid in $L$. mesenteroides subsp. mesenteroides, suggesting that it may be a plasmid with integration ability.

Another characteristic that was found to be highly variable even in close strains is chromosome size. The highest and lowest values were found in W. confusa DSM $20196^{\mathrm{T}}$ $(2197 \mathrm{~kb})$ and $W$. kandleri CECT $4307^{\mathrm{T}}(1371 \mathrm{~kb})$ respectively, which are the most extreme values ever found for Leuconostocs (Lamoureux et al., 1993; Tenreiro et al., 1994) even if they are in the range expected for $\mathrm{LAB}$ species (Klaenhammer et al., 2005). The relatively small size of LAB genomes is generally attributed to loss of genes (Makarova et al., 2006) as the result of a continuous adaptation to specific nutrient-rich environments. In fact, in the case of $L$. mesenteroides subsp. cremoris CECT $872^{\mathrm{T}}$, and to a lesser extent in the case of $W$. hellenica DSM $7378^{\mathrm{T}}$, the possible loss of genes that is translated into smaller genome sizes and chromosomal rearrangements must have been accompanied by physiological and probably ecological changes. $L$. mesenteroides subsp. cremoris is well known for its markedly reduced metabolic spectrum when compared with other $L$. mesenteroides subspecies with which it has high DNA/DNA homology (Garvie, 1983; Holzapfel \& Schilinger, 1991). In the same way, $W$. hellenica also presents a less diverse carbohydrate usage profile than $W$. paramesenteroides or $W$. confusa (Collins et al., 1993). This effect is also seen in $W$. kandleri and $W$. viridescens, whose type strains also have smaller genomes.

The type of genomic rearrangement most relevant in the chromosomes under study is undoubtedly genomic inversion. Particularly relevant in Fructobacillus and Leuconostoc, inversions like these, which involve a large proportion of the chromosome and are symmetrical with respect to oriC, are nevertheless thought to be common. Chromosomal changes of this kind have already been observed in many natural strains of Lactococcus lactis (Le Bourgeois et al., 2000), Lactobacillus (Klaenhammer et al., 2005) and even in less related genera (Eisen et al., 2000), and also in experimentally evolved strains (Campo et al., 2004). They are considered not to be very deleterious since 
both the average distance of each gene to the origin of replication and the transcription direction of most genes are largely maintained. The occurrence of inversions usually requires the presence of repeated sequences in the genome such as those from insertion sequences, prophages or multigenic families. Even if our study was not directed to finding these types of sequences we can at least say that neither $r r n$ operons nor the transposable element associated with the Transposase marker seem to have been involved in the detected inversions.

A major part of this work deals with the determination of the organization of ribosomal operons in these genomes and its possible implications. Ribosomal operons can be important elements in the evolution of chromosomal organization. Since they constitute a multigenic family with a great degree of sequence conservation they are often associated with genomic rearrangements such as duplications, deletions and inversions. In fact, although variation in $r r n$ operon copy number at the intraspecific level is uncommon (Acinas et al., 2004) it was possible to identify a duplication in Leuconostoc citreum. This event could be attributed to the type strain $L$. citreum CECT $4025^{\mathrm{T}}$ since the two other strains of this species have only four copies, which is the most frequent rrn number in Fructobacillus and Leuconostoc. Ribosomal operons seem also to be involved in, or at least are able to reveal, rearrangements in the three genera studied. A situation that appears to have resulted in the most extreme asymmetry is seen in $L$. gelidum DSM $5578^{\mathrm{T}}$, in which all the $r r n$ operons are localized to one side of the replication origin.

The analysis of the rrn operon organization enables us to distinguish strains belonging to the three groups of Leuconostoc genera: Fructobacillus plus Leuconostoc, Oenococcus and Weissella. In Fructobacillus and Leuconostoc the four rrn copies, which may be considered as the ancestral value, are clustered around oriC and always have ${ }^{\text {Ala }}$ tRNAs coded in their ITS regions. This type of ITS is also seen in both copies of the O. oeni chromosome (ZéZé et al., 2000). In this case the $r r n$ operons are usually several hundred kilobases apart, with one of them always close to oriC. In this respect, the study of the recently described Oenococcus kitaharae (Endo \& Okada, 2006) would be of great interest in order to confirm the reduced number of $r r n$ operons in the genus. Weissella is characterized by having the largest and most diverse set of $r r n$ operons. The number of $r r n$ operons varies between six and eight. This higher bound may be the result of duplications in specific Weissella groups, as can be deduced from the presence of the C8 fragment in strains of the $W$. paramesenteroides cluster. The higher number of rrn copies together with the existence of different sets of tRNAs in their ITS regions may reveal a closer proximity with other genera such as Lactococcus, Lactobacillus and Pediococcus (de Vries et al., 2005; Klaenhammer et al., 2002). As in O. oeni the origin of replication is localized in one large I-CeuI fragment although there is always a group of $r r n$ operons nearby.
It is not straightforward to provide an explanation for the relationship of these different sets of $r r n$ operons with the diversification of the three genera. If the variation in the number of $r r n$ operons reflects adaptations to different environments with different degrees of stability of resources, as was previously shown (Klappenbach et al., 2000) and proposed for $W$. paramesenteroides DSM $20288^{\mathrm{T}}$ (Chelo et al., 2004), then it is possible that the fast diversifying process proposed for these genera (Chelo et al., 2007) reflects radical changes in the ecological niches that were occupied.

Regarding the current taxonomic classification of this group, and specifically its most recent changes (Endo \& Okada, 2008), the genomic characteristics presented here support the recognition of the Fructobacillus strains as a monophyletic group. This is reflected in the general conservation of number of restriction sites, genome size and presence/absence of hybridization signals. However, it must be noted that, in what seems to be the most distinctive feature of the remaining Leuconostoc genera ( rrn number and disposition), Fructobacillus strains are very similar to Leuconostoc strains.

The physical and genetic maps described here constitute a study at different taxonomic levels that provides an analysis of the evolution of the genomes of different Leuconostoc species, enabling further insights into the evolution and diversification of the species belonging to the genera Leuconostoc, Oenococcus and Weissella.

\section{ACKNOWLEDGEMENTS}

The financial support by FCT research grants to I. M. Chelo (SFRH/ BD/10675/2002 and SFRH/BDP/27887/2006) and L. Zé-Zé (SFRH/ $\mathrm{BPD} / 3653 / 2000)$ is gratefully acknowledged.

\section{REFERENCES}

Acinas, S. G., Marcelino, L. A., Klepac-Ceraj, V. \& Polz, M. F. (2004). Divergence and redundancy of $16 \mathrm{~S}$ rRNA sequences in genomes with multiple rrn operons. J Bacteriol 186, 2629-2635.

Altschul, S. F., Gish, W., Miller, W., Myers, E. W. \& Lipman, D. J. (1990). Basic local alignment search tool. J Mol Biol 215, 403-410.

Campo, N., Dias, M. J., Daveran-Mingot, M. L., Ritzenthaler, P. \& Le Bourgeois, P. (2004). Chromosomal constraints in Gram-positive bacteria revealed by artificial inversions. Mol Microbiol 51, 511-522.

Chelo, I. M., Zé-Zé, L., Chambel, L. \& Tenreiro, R. (2004). Physical and genetic map of the Weissella paramesenteroides DSMZ $20288^{\mathrm{T}}$ chromosome and characterization of different $\mathrm{rrn}$ operons by ITS analysis. Microbiology 150, 4075-4084.

Chelo, I. M., Zé-Zé, L. \& Tenreiro, R. (2007). Congruence of evolutionary relationships inside the Leuconostoc-OenococcusWeissella clade assessed by phylogenetic analysis of the 16S rRNA gene, dnaA, gyrB, rpoC and dnaK. Int J Syst Evol Microbiol 57, 276-286.

Collins, M. D., Samelis, J., Metaxopoulos, J. \& Wallbanks, S. (1993). Taxonomic studies on some Leuconostoc-like organisms from fermented sausages: description of a new genus Weissella for the Leuconostoc paramesenteroides group of species. J Appl Bacteriol 75, 595-603. 
De Bruyne, K., Schillinger, U., Caroline, L., Boehringer, B., Cleenwerck, I., Vancanneyt, M., De Vuyst, L., Franz, M. A. P. \& Vandamme, P. (2007). Leuconostoc holzapfelii sp. nov., isolated from Ethiopian coffee fermentation and assessment of sequence analysis of housekeeping genes for delineation of Leuconostoc species. Int J Syst Evol Microbiol 57, 2952-2959.

de Vries, M. C., Siezen, R. J., Wijman, J. G. E., Zhao, Y., Kleerebezem, M., de Vos, W. M. \& Vaughan, E. E. (2005). Comparative and functional analysis of the rRNA-operons and their tRNA gene complement in different lactic acid bacteria. Syst Appl Microbiol 29, 358-367.

Eisen, J. A., Heidelberg, J. F., White, O. \& Salzberg, S. L. (2000). Evidence for symmetric chromosomal inversions around the replication origin in bacteria. Genome Biol 1, RESEARCH0011.

Endo, A. \& Okada, S. (2006). Oenococcus kitaharae sp. nov., a nonacidophilic and non-malolactic-fermenting oenococcus isolated from a composting distilled shochu residue. Int J Syst Evol Microbiol 56, 2345-2348.

Endo, A. \& Okada, S. (2008). Reclassification of the genus Leuconostoc and proposals of Fructobacillus fructosus gen. nov., comb. nov., Fructobacillus durionis comb. nov., Fructobacillus ficulneus comb. nov. and Fructobacillus pseudoficulneus comb. nov. Int J Syst Evol Microbiol 58, 2195-2205.

Garvie, E. I. (1983). Leuconostoc mesenteroides subsp. cremoris (Knudsen and Sorensen) comb. nov. and Leuconostoc mesenteroides subsp. Dextranicum (Beijerinck) comb. nov. Int J Syst Bacteriol 33, 118-119.

Holzapfel, W. H. \& Schilinger, U. (1991). The Genus Leuconostoc. In The Prokaryotes, vol. 3, pp. 1508-1533. Edited by A. Balows, H. G. Trüper, M. Dworkin, W. Harder \& K. Scheifer. Berlin: Springer.

Kandler, O. \& Weiss, N. (1986). Regular, nonsporing gram-positive rods. In Bergey's Manual of Systematic Bacteriology, vol. 2, pp. 12081234. Edited by J. G. Holt, P. H. A. Sneath, N. S. Mair \& M. E. Sharpe. Baltimore: Williams \& Wilkins.

Kim, J. F., Jeong, H., Lee, J., Choi, S., Ha, M., Hur, C., Kim, J., Lee, S., Park, H. \& other authors (2008). The complete genome sequence of Leuconostoc citreum KM20. J Bacteriol 190, 3093-3094.

Klaenhammer, T., Altermann, E., Arigoni, F., Bolotin, A., Breidt, F., Broadbent, J., Cano, R., Chaillou, S., Deutscher, J. \& other authors (2002). Discovering lactic acid bacteria by genomics. Antonie Van Leeuwenhoek 82, 29-58.

Klaenhammer, T. R., Barrangou, R., Buck, B. L., Azcarate-Peril, M. A. \& Alterman, E. (2005). Genomic features of lactic acid bacteria effecting bioprocessing and health. FEMS Microbiol Rev 29, 393-409.

Klappenbach, J. A., Dunbar, J. M. \& Schmidt, T. M. (2000). rRNA copy number reflects ecological strategies of bacteria. Appl Environ Microbiol 66, 1328-1333.
Lamoureux, M., Prévost, H., Cavin, J. F. \& Diviès, C. (1993). Recognition of Leuconostoc oenos strains by the use of DNA restriction profiles. Appl Microbiol Biotechnol 39, 547-552.

Le Bourgeois, P., Daveran-Mingot, M. \& Ritzenthaler, P. (2000). Genome plasticity among related Lactococcus strains: identification of genetic events associated with macrorestriction polymorphisms. J Bacteriol 182, 2481-2491.

Makarova, K. S. \& Koonin, E. V. (2007). Evolutionary genomics of lactic acid bacteria. J Bacteriol 189, 1199-1208.

Makarova, K., Slesarev, A., Wolf, Y., Sorokin, A., Mirkin, B., Koonin, E., Pavlov, A., Pavlova, N., Karamychev, V. \& other authors (2006). Comparative genomics of the lactic acid bacteria. Proc Natl Acad Sci U S A 103, 15611-15616.

Marcobal, A. M., Sela, D. A., Wolf, Y. I., Makarova, K. S. \& Mills, D. A. (2007). The role of hypermutability in the evolution of the genus Oenococcus. J Bacteriol 190, 564-570.

Martinez-Murcia, A. J. \& Collins, M. D. (1990). A phylogenetic analysis of the genus Leuconostoc based on reverse transcriptase sequencing of 16S rRNA. FEMS Microbiol Lett 58, 73-84.

Pitcher, D. G., Saunders, N. A. \& Owen, R. J. (1989). Rapid extraction of bacterial genomic DNA with guanidium thiocyanate. Lett Appl Microbiol 8, 151-156.

Tenreiro, R., Santos, M. A., Paveia, H. \& Vieira, G. (1994). Inter-strain relationships among wine leuconostocs and their divergence from other Leuconostoc species, as revealed by low frequency restriction fragment analysis of genomic DNA. J Appl Bacteriol 77, 271-280.

Vandamme, P., Pot, B., Gillis, M., De Vos, P., Kersters, K. \& Swings, J. (1996). Polyphasic taxonomy, a consensus approach to bacterial systematics. Microbiol Rev 60, 407-438.

Yang, D. \& Woese, C. R. (1989). Phylogenetic structure of the "Leuconostocs": an interesting case of a rapidly evolving organism. Syst Appl Microbiol 12, 145-149.

Zé-Zé, L., Tenreiro, R., Brito, L., Santos, M. \& Paveia, H. (1998). Physical map of the genome of Oenococcus oeni PSU-1 and localization of genetic markers. Microbiology 144, 1145-1156.

Zé-Zé, L., Tenreiro, R. \& Paveia, H. (2000). The Oenococcus oeni genome: physical and genetic mapping of strain GM and comparison with the genome of a 'divergent' strain, PSU-1. Microbiology 146, 3195-3204.

Zé-Zé, L., Chelo, I. M. \& Tenreiro, R. (2008). Genome organization in Oenococus strains studied by comparison of physical and genetic maps. Int Microbiol 11, 237-244.

Edited by: H.-P. Klenk 